\title{
The Effect of Music Therapy on the Level of Blood Cortisol in Pregnant Women Conceived by In Vitro Fertilization (IVF) Technology
}

\section{Satish Patki ${ }^{1 *}$, Rajendra Patil ${ }^{2}$ and Shweta Patki Kulkarni ${ }^{1}$}

${ }^{1}$ Department of Obstetrics and Gynecology, IVF Consultant, Patki Hospital and Research Foundation, Post Graduate Institute of Obstetrics and Gynecology,

Kolhapur, India

${ }^{2}$ Department of Pathology, Consultant Pathologist, Patki Hospital and Research Foundation, Post Graduate Institute of Obstetrics and Gynecology, Kolhapur, India

*Corresponding Author: Satish Patki, Department of Obstetrics and Gynecology, IVF Consultant, Patki Hospital and Research Foundation, Post Graduate Institute of Obstetrics and Gynecology, Kolhapur, India.
Received: February 20, 2020

Published: March 20, 2020

(C) All rights are reserved by Satish Patki., et al.

\begin{abstract}
Introduction: In vitro fertilization (IVF) is a modern technology for the treatment of infertile couples. However, in women with pregnancies achieved using IVF technique, the stress levels are higher. Blood cortisol levels are elevated in stressful situations. Music has a potential of generating positive feelings and "medicinal music" has gained popularity especially in pregnant women. Hence, in addition to the conventional protocol based treatment to such women, music therapy can be considered as a complementary treatment to reduce the stress and to get better clinical outcomes. Present study is designed to scientifically evaluate the role of music therapy in women with pregnancy achieved through IVF technology.

Aims and Objectives: To study the effect of music therapy on blood cortisol level in pregnant women conceived by IVF technique Materials and Methods: 30 pregnant women conceived by IVF with gestation more than 20 weeks were selected. After sensitizing them with three sessions of music therapy with a 20 minutes audio track of old Hindi film songs, they were subjected for the study. Pre and 30 minute post music therapy of listening the same audio track in the morning hours, the blood cortisol levels were estimated.

Results: As the patients achieved pregnancy through IVF, $26.67 \%$ of them were from elderly and perimenopausal age group. $73.34 \%$ patients had long standing infertility more than 10 years. The serum cortisol levels were found to be significantly reduced from mean $17.80 \mathrm{mcg} / \mathrm{dl}$ to $14.73 \mathrm{mcg} / \mathrm{dl}$ ( $\mathrm{p}$ value $<0.05$ ) after music therapy.

Conclusions: Music therapy can be used as a complementary treatment in women with IVF pregnancies to reduce the stress and associated raised blood cortisol levels to get good clinical maternal and perinatal outcome.
\end{abstract}

Keywords: In Vitro fertilization (IVF); Music Therapy; Cortisol Levels; Stress; Pregnancy Induced Hypertension

\section{Abbreviations}

IVF: In Vitro Fertilization; PIH : Pregnancy Induced Hypertension; GDM: Gestational Diabetes Mellitus.

\section{Introduction}

In vitro fertilization (IVF) is the modern technology to achieve pregnancy in infertile couples where conventional medical treat- ment fails. The incidence of in vitro fertilization (IVF) is steadily increasing. These are patients with prolonged infertility, often older and perimenopausal. Many of them have undergone gynecologic surgery and have extragenital pathology. However the treatment involves many procedures which themselves induce stress to the couple especially to the wife. Daily injections, blood draws and 
tests, ultrasound examinations, oocyte retrieval, laboratory results of embryo formation and finally the result of the procedure of embryo transfer leading to its implantation and the successful pregnancy. Oocyte Retrieval and result of pregnancy test constitute the most stressful stages of IVF cycle [1-3].

Successful pregnancy after IVF procedure though gives joy to the couple, actually initiates the stress during the course of such pregnancy. Often after IVF there is a multiple pregnancy, which is accompanied by a complicated course. The fear of abortion, preterm delivery, congenital fetal anomalies and other medical as well as surgical problems including complications of caesarean section evokes continuous and chronic stress during such IVF pregnancy. The stress created during the pregnancy is known to induce the diseases like pregnancy induced hypertension (P.I.H.) Gestational diabetes mellitus (GDM) in such women.

Music is a non pharmacological modality which is non invasive, non medical with no known side effects, cost effective and easily accessible to all the socioeconomic groups of the patients. Music heals the soul and also influences immune and endocrine function [4]. History reveals that the traditional Indian healing systems involve vedic chants and music. In the last two decades, the concepts of "medicinal music" has gained increasing popularity and one of the most widely known applications of music in the medical field has been audio analgesia [4].

In the $20^{\text {th }}$ century, the publication of articles of research on the therapeutic effectiveness of music in the field of obstetrics has shown that music during pregnancy promotes wellbeing of the mother and fetus, increases relaxation and provides calming effect and alleviates stress [5].

It is an established fact that during the periods of stress there is dysregulation of hypothalamic- pituitary- adrenal(HPA) axis leading to raised levels of serum cortisol [6]. Therefore, study of serum cortisol levels in women conceived by IVF technology before and after listening music is the main focus of the present study.

\section{Materials and Methods}

Present study was carried out at Patki hospital and Research Foundation, a Postgraduate Institute of Obstetrics and Gynecology, Kolhapur, India during the period from August 2019 To January 2020 . The study was approved by the ethics committee. Thir- ty patients conceived by IVF technology with the gestation of 20 weeks and more were selected randomly. Patients having acute or chronic hormonal dysregulations including thyroid disorders were excluded. The patients with psychiatric illness were also excluded. Informed and written consent was obtained from the patient and her husband. The patients were on the conventional protocol based antenatal treatment and regular follow up. In addition to conventional medical treatment, these patients were offered the option of music therapy as a supportive treatment.

To study the effects of the music, the musical track of twenty minutes duration was prepared. The track consisted of the old songs from Hindi films made under the direction of famous musicians Laxmikant Pyarelal.

The track was loaded on the music player of the cellphone of the patient. Detailed counselling of the couple was done by the clinician and medical counselor about the instructions of how to listen the music. The patient was asked to listen the track alone in the morning hours (between 8 and $9 \mathrm{am}$ ) by using earphones in a separate room with no other disturbances. After three sessions on three different days, each patient was subjected for the cortisol study with music therapy. The pretest venous blood sample for serum cortisol estimation was obtained. Each patient was asked to listen the musical track for 20 minutes and 30 minutes after the music therapy session, the post test venous blood sample was collected for serum cortisol estimation. The blood was immediately centrifuged for 10 minutes and the supernatant plasma was subjected for the serum cortisol estimation in micrograms per deciliter using chemiluminescence assay.

The data obtained from all the subjects was tabulated into the master data sheet.

\section{Statistical analysis}

The data was entered into Microsoft excel data sheet and was analyzed using SPSS 22 version software Continuous data was represented as mean and standard deviation. Independent paired $t$ test was used as a test of significance to identify the mean difference between two quantitative variables. Graphical representation of the data was done in MS excel and MS word. P value of 0.05 was considered as statistically significant after assuming all the rules of statistical tests. 
The Effect of Music Therapy on the Level of Blood Cortisol in Pregnant Women Conceived by In Vitro Fertilization (IVF) Technology

\section{Results}

Total number of subjects selected were 30 , all with IVF pregnancies.

\begin{tabular}{|l|c|c|}
\hline Age (In Years) & Number of Patients & Percentage \\
\hline $20-30$ & 9 & $30.00 \%$ \\
\hline $30-40$ & 13 & $43.33 \%$ \\
\hline $40-50$ & 8 & $26.67 \%$ \\
\hline Total & 30 & $100.00 \%$ \\
\hline
\end{tabular}

Table 1: Age wise distribution.

Majority of the patients were of the age ranges of 30-40 years. IVF technology is successful in elderly and even perimenopausal patients, hence $26.67 \%$ patients in the present study were above the age of 40 years.

\begin{tabular}{|l|c|c|}
\hline $\begin{array}{l}\text { Duration of Married } \\
\text { Life( In Years) }\end{array}$ & $\begin{array}{c}\text { Number of } \\
\text { Patients }\end{array}$ & Percentage \\
\hline $5-10$ & 8 & $26.66 \%$ \\
\hline $10-15$ & 11 & $36.66 \%$ \\
\hline $15-20$ & 9 & $30.02 \%$ \\
\hline $20-25$ & 1 & $3.33 \%$ \\
\hline $25-30$ & 1 & $3.33 \%$ \\
\hline Total & 30 & $100.00 \%$ \\
\hline
\end{tabular}

Table 2: Duration of Infertility.

All the patients had infertility and all had earlier medical treatment and had finally opted for IVF to conceive. Though majority of the patients had married life duration of 5 to 15 years, $36.66 \%$ had infertility of more than 15 years.

$70 \%$ of the patients had pregnancy induced hypertension (P.I.H.) and were under medical treatment. Two patients had gestational diabetes and were on insulin treatment.

\begin{tabular}{|l|c|c|}
\hline Weeks of Gestation & Number of Patients & Percentage \\
\hline 20 to 25 & 4 & $13.33 \%$ \\
\hline 25 to 30 & 7 & $23.33 \%$ \\
\hline 30 to 35 & 10 & $33.33 \%$ \\
\hline 35 to 40 & 9 & $30.01 \%$ \\
\hline Total & 30 & $100.00 \%$ \\
\hline
\end{tabular}

Table 3: Gestational age wise distribution.

\begin{tabular}{|l|c|c|}
\hline Number of Fetuses & Number of Patients & Percentage \\
\hline Singleton & 16 & $53.33 \%$ \\
\hline Twins & 13 & $43.33 \%$ \\
\hline Triplets & 1 & $3.34 \%$ \\
\hline Total & 30 & $100.00 \%$ \\
\hline
\end{tabular}

Table 4: Number of fetuses.

In one patient having twin pregnancy one fetus died in utero at 30 weeks due to P.I.H. However the patient was under observation and the pregnancy was continued till 35 weeks. In one patient with triplets, there was major placenta previa which used to lead to frequent episodes of painless and causeless vaginal bleeding. Both the above patients were taking music therapy and finally delivered viable babies at 35 weeks of gestation.

\begin{tabular}{|l|c|c|}
\hline Parameters & $\begin{array}{c}\text { Before Music } \\
\text { Therapy }\end{array}$ & $\begin{array}{c}\text { After Music } \\
\text { Therapy }\end{array}$ \\
\hline Mean $(\mathrm{mcg} / \mathrm{dl})$ & 17.80 & 14.73 \\
\hline Std. Deviation $(\mathrm{mcg} / \mathrm{dl})$ & 21.18 & 18.26 \\
\hline Std. Error Mean & 3.87 & 3.33 \\
\hline Mean Difference $(\mathrm{mcg} / \mathrm{dl})$ & \multicolumn{2}{|c|}{3.04} \\
\hline T Value & \multicolumn{2}{|c|}{3.30} \\
\hline P Value & \multicolumn{2}{|c|}{0.002} \\
\hline
\end{tabular}

Table 5: Serum cortisol levels before and after music therapy.

In the present study, after giving music therapy in 30 Patients, it was found that serum cortisol levels significantly reduced from mean $17.80 \mathrm{mcg} / \mathrm{dl}$ to $14.73 \mathrm{mcg} / \mathrm{dl}$ (P value < 0.05$)$ depicting that music therapy is quite effective in reducing serum cortisol levels.

Effect of music therapy on mean cortisol levels (mcg/dl)

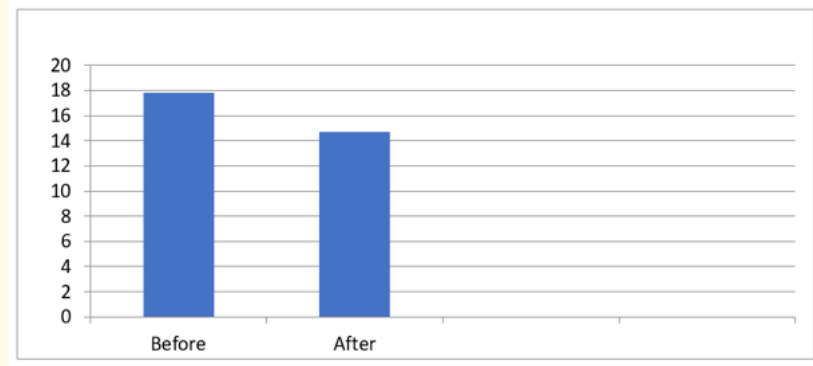

Figure 


\section{Discussion}

It is an established fact that IVF pregnancy is associated with a lot of stress. In fact the patient starts developing the stress from the day when she actually starts IVF treatment. Many of patients are elderly. In the present study among the patients of the main cohort, $26.67 \%$ patients were of perimenopausal age group. The duration of infertility is also a factor which is directly proportional to the level of stress. In the present study $36.66 \%$ patients had infertility of duration of 10 to 15 years while $36.68 \%$ patients had very long standing infertility of duration of 15 to 30 years. During this period they had undergone many invasive diagnostic procedures like endoscopic surgery, hysterosalpingographies, endometrial biopsies and sonographies. They had a lot of psychological social pressure from their relatives, neighbors and colleagues. In a country like India, the female partners are under continuous mental tension when they are not conceiving. IVF pregnancies are many a times multifetal. In present study $46.67 \%$ patients had multifetal pregnancies. Such multifetal pregnancies are often associated with complications like pregnancy induced hypertension (P.I.H.) Gestational diabetes mellitus (GDM), preterm labour, antepartum hemorrhage etc. Thus, elderly age group, prolonged infertility, IVF conception and frequent association of multifetal pregnancies are the stress evoking factors in such patients. Obviously, the stress level in all the patients of the study was high. Such patients require individualized treatment and observation according to the principles of personal medicine. Reducing stress levels for these patients in an important aspect of having a successful outcome of pregnancy.

The axis of hypothalamus-pituitary-adrenal (HPA) is activated in response to the stress. The increase in the level of stress and anxiety can affect the function of this axis and leads to increase in the level of cortisol in blood [7]. Cortisol is a glucocorticoid secreted by adrenal cortex which is also named as stress hormone [8]. The rise in the level of cortisol causes the rise in the level of blood glucose and insulin resistance. The high level of blood glucose has undesirable effects on the process of wound recovery which has a special significance for the patients undergoing surgical operation. Cortisol also increases blood pressure by raising the arterial tone. A high level of cortisol induces inflammation process and reduces immunity [9].

High levels of stress also stimulates sympathetic nervous system resulting in increased levels of norepinephrine leading to tachycardia and increased respiratory rate. Stress also affects cytokines, lymphocytes and immunoglobulins. In the present study we have focused on the cortisol levels as an indicator of stress.

Recorded music is a non-medicinal nursing interference which is a non-expensive and is without any harmful effects. It can be used as a complementary method in lessening the stress and the anxiety of the patients. Many studies like Stefano., et al. [10], Allred., et al. [11] and Han., et al. [12] have shown the efficacy of music therapy in reducing the anxiety and stress.

The study of effect of music therapy on blood cortisol levels has been a topic of research for last many decades in various fields of medicine. There are many studies proving the beneficial role of music therapy during surgeries, angiographies and colonoscopies. Shivamurthy., et al. [13] has studied the effect of music therapy during active labour. In this study the patients during active labour pains were given music therapy and their serum cortisol levels were studied before and after music therapy. The study proved statistically significant difference in blood cortisol levels before and after music therapy. E laheh., et al. [14] have also studied serum cortisol levels before and after music therapy in patients undergoing surgical procedures and found significant difference in pre and post music therapy groups.

Stress and its effect on cortisol level has been also a topic of research in IVF patients. Yuan., et al. [15] have shown the relationship between stress, elevated blood cortisol levels and their effect on pregnancy rate as well as live birth rate in IVF patients. However, there is no study on the effects of music therapy on cortisol levels and clinical outcome in IVF patients. This is the first of its kind study on the effects of music therapy on cortisol levels in pregnant patients conceived with IVF.

Blood cortisol levels are known for their wide fluctuations and diurnal variation in the same individual. Hence, in the present study patients were given music therapy in the morning hours preferably at the same time slot. It was found that serum cortisol levels were significantly reduced from mean $17.80 \mathrm{mcg} / \mathrm{dl}$ to $14.73 \mathrm{mcg} / \mathrm{dl}$ (p value $<0.05$ ) after giving the music therapy. Music used in our study was in the form of old Hindi film songs which are capable of generating positive feelings and lifting patients spirits. Two patients with very high risk pregnancy (one with triplets and placenta previa and 
other with twin with one dead fetus) were highly stressed. They showed marked reduction in their serum cortisol levels after music therapy. They followed the music therapy throughout antenatal period after 24 weeks of gestation and could successfully delivered viable babies.

\section{Conclusion}

The results of the present study reveal that music therapy can be used as a useful complement to the basic algorithm to reduce the stress and its associated pathophysiological effects like raised cortisol levels in the pregnant women with higher stress levels due to factors associated with IVF conception. This can lead to good maternal and perinatal outcome of such high risk pregnancies.

\section{Limitation of the Present Study}

This is our initial successful experience of music therapy in stressed moms. There is a need to increase the sample size for further valid conclusions and to formulate clinical recommendations. Apart from serum cortisol levels the other clinical parameters of stress like heart rate, blood pressure and respiratory rate were not evaluated in the present study. Similarly other biochemical parameters like estimation of cytokines and norepinephrine and salivary amylase levels were also not studied. Final clinical outcome of the mother and neonate should also be followed in larger number of patients. A comprehensive study inclusive of all the parameters will prove the role of music therapy further.

\section{Acknowledgements}

We would like to express our gratitude to the legendary figure in the field of Hindi film music Mr. Pyarelalji Sharma of the duo Laxmikant Pyarelal for taking special interest in this research. We are also thankful to Mrs. Sunilaji Pyarelal Sharma, Mr. Gautam Pyarelal Sharma and Mr. Vivek Patki for preparing the music track used for the music therapy of the present project.

\section{Conflict of Interest}

We declare that there are no conflicts of interest in this research project. There is also no financial support for the project.

\section{Bibliography}

1. Yong P., et al. "A comparison of psychological functioning in women at different stages of in vitro fertilization treatment using the mean affect adjective checklist". Journal of Assisted Reproduction and Genetics 17 (2000): 553-556.
2. Kolonoff-Cohen H., et al. "A Prospective study of stress among women undergoing in vitro fertilization or gamete intrafallopian transfer". Fertility and Sterility 76 (2001): 675-687.

3. Bovin J., et al. "Reactions to infertility based on extent of treatment failures". Fertility and Sterility 63 (1995): 801-807.

4. "Music and audio analgesia section B: clinical guidelines for obstetrics and midwifery" (2007).

5. Phumodoung S and Marion G. "Music reduces sensation and distress of labour pain”. Pain Management Nursing 4 (2003) 54-61.

6. Kolonoff-Cohen $\mathrm{H}$ and Natarajan L. "The concerns during assisted reproductive technologies scale and pregnancy outcomes". Fertility and Sterility 81 (2004): 982-988.

7. Kudielka., et al. "HPA axis responses to laboratory psychosocial stress in healthy elderly adults, younger adults and children". Psychoneuroendocrinology 29 (2004): 83-98.

8. Koelch S., et al. "Effects of music listening on cortisol levels and propofol consumption during spinal anesthesia". Frontier Psychology 2 (2011): 58.

9. Asghaari H and Lotfi Mozhgan A. "Effect of preoperative nursing visiton serum cortisol levels in patients waiting for operation: a randomized study with control group". Medical Journal of Tabriz University of Medical Sciences 30 (2009): 13-16.

10. Stefano G., et al. "Music alters constitutively expressed opiate and cytokine processes in listener". Medical Science Monitor (2004): 10-18.

11. Allred K D., et al. "The effect of music on postoperative pain and anxiety". Pain Management Nursing 11 (1010): 15-25.

12. Han., et al. "Effects of music intervention on physiological stress response and anxiety level of mechanically ventilated patients in china: a randomized controlled trial". Journal of Clinical Nursing 19 (2010): 978-987.

13. Shivamurthy G and Anusha G. "Effect of music therapy on serum cortisol in primigravida in active labour". IOSR Journal of Dental and Medical Sciences 15 (2016): 91-94.

14. Elaheh M., et al. "The effect of music on the level of cortisol, blood glucose and physiological variables in patients undergoing spinal anesthesia”. EXCL Journal 11 (2012): 556-565. 
15. Yuan A., et al. "Relationship between psychological stress and reproductive outcome in women undergoing in vitro fertilization treatment: psychological and neurohormonal assessment". Journal of Assisted Reproduction and Genetics 3030 (2013): 35-41.

\section{Assets from publication with us}

- Prompt Acknowledgement after receiving the article

- Thorough Double blinded peer review

- Rapid Publication

- Issue of Publication Certificate

- High visibility of your Published work

Website: www.actascientific.com/

Submit Article: www.actascientific.com/submission.php

Emaill us: editor@actascientific.com

Contact us: +919182824667 